\title{
Patient Preparation for 18 F FDG PET-CT scan - A Short Communication
}

Fatima Begum, Raihan Hussain, Shamim Momtaz Ferdousi Begum and Mizanul Hasan

National Institute of Nuclear Medicine and Allied Sciences.

Correspondence Address : Prof. Dr. Fatima Begum, National Institute of Nuclear Medicine \& Allied Sciences, BAEC, Shahbagh, Dhaka, Bangladesh, Email: fatima8ninmas@gmail.com

\section{INTRODUCTION}

18-F fluorodeoxyglucose Positron Emission Tomography- Computed Tomography (18F FDG PETCT) scan is an emerging modality of imaging in developing country like us although it is an expensive one. This sophisticated imaging is now available for the general people of Bangladesh, for which a good number of patients used to go to the neighboring countries in previous years. We are proud to say that we are going to stop this trend. Most of the general physicians are eager to be acquainted with this procedure. 18 F FDG PETCT scan play an important role mostly in oncology, less commonly in neurology and cardiology and diagnosis of inflammatory diseases. In oncology, this imaging is useful in diagnosis of primary site of cancer, staging, restaging after treatment, radiation therapy planning and to see the prognosis after chemotherapy and radiotherapy (1). Several factors affect the 18 F FDG PET-CT scan. To get good quality image adequate patient preparation is essential. This article is intended to address the challenges to prepare patients for PETCT scan especially in diabetic patients and other conditions and to disseminate this information to all physicians as well as patients in simple language.

When patients are referred to Nuclear Medicine Department, physicians are to evaluate the feasibility of doing PET-CT scan. 18 F FDG PET-CT scan cannot be done instantly on each patient. We need to be careful about timing for this scan considering other relevant events of patients. Usually 18 F FDG PET-CT scan should be scheduled as follows- post biopsy- 1 week; post surgery- 6 weeks; post radiofrequency ablation-4 weeks, post chemotherapy-2-4 weeks, post radiation-
2-6 months, and post granulocyte colony-stimulating factor-1 week to1 month $(2,3)$.

Careful patient preparation is essential to get good quality images, which are intended for interpretation of PET-CT studies. Before study procedure physicians are to consider different aspects which have impact on PET-CT scan- those are restrictions of diet, exercise/activity and awareness of medications and environment factors. Blood glucose level has significant role in 18 F FDG uptake as 18 F FDG and glucose compete for glucose transport and phosphorylation (2). During pregnancy PET-CT is contraindicated and they are also discouraged to attend the patients. Patients would expect 2-4 hours time to spend in Nuclear Medicine Department to complete all segments of test including waiting time. 18 FDG is to be administered intravenously after taking rest for an hour in quiet comfortable room. Scan time is usually 20-25 minutes. There are several guidelines and published data for PET-CT scan procedures including patient preparation. Those are based on scientific experiences, but there is little variation between guidelines and institutional procedures due to different settings. The following instructions has been adopted from those guidelines and published data which are feasible with our culture and habits of our population.

Instructions about diet: Patients are instructed fasting for at least 6 hours $(1,3)$ and in some cases fasting for 4 hours may be allowed (4). To study the thoracic pathology 12 hours fasting is recommended to minimize cardiac activity (1). Low carbohydrate and high protein/ fat diet prior examination day for at least 24 hours can optimize FDG uptake and decrease myocardial uptake. Avoidance of rice and bread in 
previous day and day of examination has been mentioned in most of the guidelines and institutional pamphlet. We have recommended low carbohydrate diet-lowest possible amount of rice at noon in previous day and single piece of homemade bread at night with adequate amount of protein (meat, egg). Any kind of sugar, sweetmeat and sweet fruits are to be avoided in previous day and day of study. This preparation worked in patients of our department. Blood glucose should be checked in all patients prior to FDG injection. Recommended glucose level by Society of Nuclear Medicine and Molecular Imaging (SNMMI) is between $7 \mathrm{mmol} / \mathrm{L}(120 \mathrm{mg} / \mathrm{dl})$ and 11 $\mathrm{mmol} / \mathrm{L}(200 \mathrm{mg} / \mathrm{dl})$ (4). European Association of Nuclear Medicine (EANM) recommended glucose level below $7 \mathrm{mmol} / \mathrm{L}$ (120 mg/dl) (3) for PET study.

Water: Patients can take water as much as they can in previous day (6 glasses/1.5 L) and can take only water on the day of scan for good hydration. Tea, coffee, any caffeine containing drink, juice, alcohol and milk are to be avoided for 24 hours.

In diabetic patients: Patients are encouraged to frequent home blood glucose checks in previous days of the study. Diabetic patients should take low carbohydrate diet in previous day and patient should come in fasting condition, if appointed in early hour. All oral antidiabetic drugs can be taken as directed in prescriptions in prior day. Tablet metformin can be avoided on the date of exam and previous day in cases of colonic cancer patients-these drugs enhance colonic uptake which can hide lesions. If scan time is scheduled later in day, patient can take light breakfast with mainly protein and can take insulin injection (long acting in early morning about 6 AM). Short acting insulin (2-5 units) may be given one and one and half hours of FDG injection to reduce blood glucose level $(1,2)$.

Physical activity and exercise: Patients should avoid strenuous exercise, household work, walking, sexual activity, chew gum, betel leaves or nuts and jogging etc for previous 24 hours. If patients do not take rest, skeletal muscles take more FDG.
Environmental factor: Patients are to avoid cold exposure for 2 days before study. They are to be kept in warm environment before FDG injection-they are covered by blanket in winter, otherwise there will be brown fat uptake.

Medication: Patients are advised to take all other medications as per prescriptions in previous day and day exam with water only.

Talking: Patient should not talk 5 min before and after FDG injection until scan time and take rest quietly, otherwise, FDG will accumulate in vocal cords (laryngeal muscles) $(1,2)$

Clothes: Patients are to wear comfortable dressessweat pant and sweatshirt (cotton). For CT metallic object (zipper, button and hook) should be avoided.

Patients with colostomy bag: Patients with colostomy/ileostomy bags should come with clean bag at morning. Furthermore, they are advised to bring a new bag with attendant who will change the bag before scanning (5).

Post scan advice: After completion of PET-CT scan patients are advised to take plenty of water and void bladder frequently. They can resume their usual work on same day if not barred otherwise.

\section{REFERENCES}

1. Devaki Shilpa Surasi, Pradeep Bhambhvani, John A. Baldwin, Samuel E Almodorvar and Janis P O' Malley. 18F- FDG PET and PET/CT Patient Preparation: A review of Literature. J NUcl Med Technol 2014;42:5-13

2. Eugene $\mathrm{C}$ Lin and Abass Alavi. Patient Preparation.In: Eugene $\mathrm{C}$ Lin and Abass Alavi, eds. PET and PET/CT a Clinical Guide. 2 nd Ed, Thieme Medical Publishers, Inc. New York/ China;2009:33-37.

3. Boellaard R, O' Doherty MJ, Weber WA et al. FDG PET and PET/CT: EANM Procedure Guidelines for Tumor PET Imaging- version1.0. Eur J Nucl Mol Imaging 2010;37:181200.

4. Delbeke D, Coleman RE, Guiberteau MJ, et al. Procedure Guideline for Tumor Imaging with 18 F-FDG PET/CT 1.0. J Nucl Med 2006;47:885-895.

5. Sharon $\mathrm{M}$ Hamblen, Val J Lowe. Clinical 18 F-FDG Oncology Patient Preparation Techniques. J Nucl Med Technol 2003;31:3-10. 